\title{
Flowering plant phylogeny and soil to plant transfer of radionuclides
}

\author{
N. Willey and K. Fawcett \\ Centre for Research in Plant Science, University of the West of England, \\ Coldharbour Lane, Frenchay, Bristol BS16 1QY, U.K.
}

\begin{abstract}
.
Our understanding of the evolutionary relationships (phylogenies) between flowering plants has been transformed in the last yen years. As a result, new phylogenies for flowering plants have been developed specifically for comparative biological studies. Researchers have used these new phylogenies to help explain variation in a number of plant characteristics. We have used them to help explain soil to plant transfer of heavy metal ions and have recently finished applying them to studies of soil to plant transfer of radionuclides.

Using REML analyses we combined literature data and data from our own experiments to provide data bases of relative concentrations of ${ }^{137} \mathrm{Cs},{ }^{60} \mathrm{Co},{ }^{15} \mathrm{~S},{ }^{131} \mathrm{l},{ }^{90} \mathrm{Sr},{ }^{36} \mathrm{Cl}$ and ${ }^{109} \mathrm{Ru}$ in up to 200 plant taxa. Analysis of these databases using heirarchical ANOVAs showed that radionuclide concentration in plants is significantly affected by phylogeny. There are some similarities and differences in phylogenetic patterns between the different radionuclides. In particular, for many radionuclides concentrations in monocotyledonous plants are significantly lower than for other flowering plants. Certain plant orders including the Caryophyllales and Cucurbitales have a significant tendancy to accumulate radionuclides. The implications of these findings for understanding and predicting soil to plant transfer of radionuclides in a wide range of plant taxa will be discussed.
\end{abstract}

\section{INTRODUCTION}

\subsection{New Phylogenies}

In the last ten years the emergence of large scale gene-sequencing ability and increasingly sophisticated computer software have combined to transform our understanding of the evolutionary relationships (phylogenies) between living organisms. Sophisticated phylogenetic techniques are now routinely used to investigate the relationships between living organisms. An international consortium of scientists. The Angiosperm Phylogeny Group, have used these techniques to investigate the relationships between flowering plants. They proposed a new phylogeny for flowering plants, based on molecular and other phenotypic data, that differs in important respects from previous phylogenies [1]. This new understanding of the phylogeny of flowering plants has been explicitly formulated to be useful to comparative biological studies [2]. One set of comparative biological studies to which it is relevant is soil to plant transfer of radionuclides by different species of plants.

The new phylogeny for flowering plants [1-2] is significantly different to previous classification systems in the fundanental divisions of flowering plants. There is no simple divide into monocotyledonous and dicotyledonous classes. Rather, as shown in figure 1 , cotyledon number is now widely acknowledged not to be a primary taxonomic character and there are a number of primitive groups of plants with living relatives, out of which the monocotyledons evolved. The monocots therefore remain as a highly distinct clade but the dicots have been split into the primitive plant clades and the eudicot clade, based not on cotyledon number but on pollen morphology. There are a number of primitive core eudicot clades with living relatives, primarily the Caryophyllids whilst the most recently evolved eudicots split into the Rosids (with Eurosid 1+2) and Asterids (with Euasterids 1+2). 


\subsection{New Phylogenies and Plant Biology}

The new phylogeny of flowering plants has been used to clarify our understanding of variation in a number of important plant characteristics. For example, in previous phylogenies of flowering plants the ability to form nitrogen fixing symbioses with micro-organisms seemed to be quite widely distributed taxonomically. This implied that the propensity to form nitrogen fixing symbioses had evolved numetous times in flowering plants. However, when plant species that form nitrogen fixing symbioses are mapped on to the new phylogeny all those plants that form them lie on a single branch of the evolutionary tree [3]. This implies that the ability to form nitrogen fixing symbioses may only have evolved once in flowering plants. Such research has improved significantly our understanding of the nitrogen symbiotic characteristics of plants and has now been applied in a number of areas of plant biology.

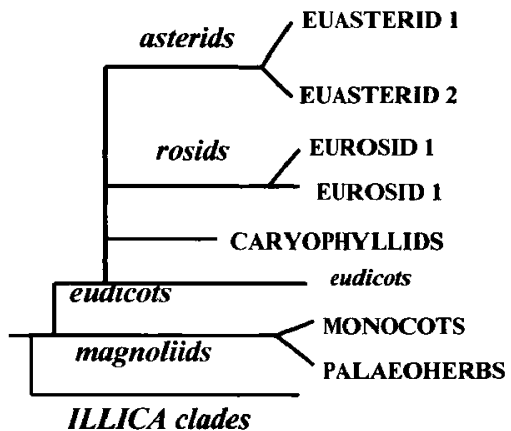

Figure 1. A Generalised outline of the phylogeny of flowering plants [2].

\subsection{Phylogeny and Plant Uptake of Radionuclides}

We have recently shown that variation in $\mathrm{Zn}, \mathrm{Cd}, \mathrm{Cu}, \mathrm{Ni}, \mathrm{Pb}$ and $\mathrm{Co}$ is significantly affected by phylogeny [4]. When the new phylogeny for flowering plants [2] is used it is clear that for these metals there are certain groups of plants that have either high or low uptake for particular metal ions. This proved to be an interesting observation for a number of reasons. First, it enables predictions of soil to plant transfer of metal ions to be made. Importantly, because groups of plants are identified, it is possible to make predictions for plant taxa in which there have been no investigations of their uptake characteristics. Second, it enables hypotheses to be formulated about the plant mechanisms that might underpin soil to plant transfer. And. third it enables a quantification of the biodiversity of response in soil to plant transfer that exists in plants. In this paper, we outline our progress in applying the new phylogeny for flowering plants to understanding soil to plant transfer of ${ }^{137} \mathrm{Cs},{ }^{60} \mathrm{Co},{ }^{35} \mathrm{~S},{ }^{131} \mathrm{I},{ }^{90} \mathrm{Sr},{ }^{36} \mathrm{Cl}$ and ${ }^{109} \mathrm{Ru}$. The results have implications for understanding 1 . soil to plant transfer of these radionuclides, 
2. the mechaisms that control their uptake, and 3. The biodiversity available for managing soil to plant transfer of radionuclides.

\section{METHODS}

\subsection{Databases}

In order to investigate the effect of phylogeny of soil to plant transfer of radionuclides large databases of radionuclide concentrations in plants are necessary. We have developed a method based on Residuat Maximum Likelihood analysis for constructing such databases [5]. The method relativises logtransformed plant concentrations across different data sets if the data sets have species in common. It can, therefore, be used to construct data bases from previously existing data sets. However, existing data sets may not have taxa in common and may also be quite restricted in the taxa they include (for example, many existing data sets are heavily biased towards certain food crops). We therefore generate are own data sets using taxa that can link existing data sets together and give taxonomic spread to the database.

The databases provide concentrations of radionuclides in shoot material in relative units. The REML procedure treats each data set in the data base as an experimental block and in effect removes the influence of factors such as soil availability. This assumes that the effect of environmental variables, such soil availability, on the rank order of radionuclide concentrations is always the same. This may not be the case. This is a confounding influence that might obscure some phyloenetic effects but that we currently have no method to deal with. When databases of relative concentrations of radionuclides have been compiled the data is analysed using a nested hierarchical Analysis of Variance. The categories used for this ANOVA are derived from the new phylogeny of flowering plants [2]. This analysis apportions sums of squares to each level of the taxonomic heirarchy and generates mean values for each category at each level.

\section{RESULTS}

The results of the REML and ANOVA show that for many radionuclides there is a large and significant

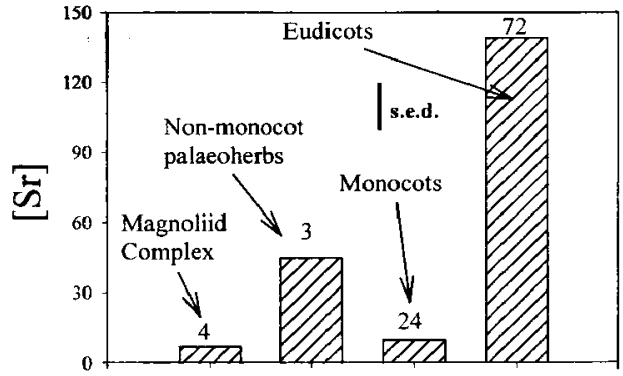

Figure 2. Mean relative concentrations of ${ }^{\text {s.0 }} \mathrm{Sr}$ in flowering plant classes. (XX=number of taxa; s.e.d = standard error of the difference between monocots and eudicots). 
difference between the radionuclide concentrations in primitive angiosperm clades, the monocot clade and the eudicot clade. For example, in the case of ${ }^{90} \mathrm{Sr}$ there is a highly significant difference between the monocot and eudicot clades (Figure 2).

We have found similar patterns in the uptake of ${ }^{137} \mathrm{Cs}[5],{ }^{36} \mathrm{Cl},{ }^{109} \mathrm{Ru}$, and ${ }^{60} \mathrm{Co}[4]$. Lower down the taxonomic heirarchy there are significant differences between Orders of plants in their radionuclide burdens. Table 1 shows those plant orders that the results suggest have unusual uptake of radionuclides.

Table 1. Plant Orders With Unusual Radionuclide Uptake Characteristics.

\begin{tabular}{|c|c|c|}
\hline Radioisotope & $\begin{array}{l}\text { Plant Orders With } \\
\text { High Uptake }\end{array}$ & $\begin{array}{l}\text { Plant Orders With } \\
\text { Low Uptake }\end{array}$ \\
\hline${ }^{137} \mathrm{Cs}$ & Caryophyllales & Poales \\
\hline${ }^{60} \mathrm{Co}$ & Caryophyllales & Poales \\
\hline${ }^{109} \mathrm{Ru}$ & Cucurbitales & Poales \\
\hline${ }^{90} \mathrm{Sr}$ & Cucurbitales & Poales \\
\hline
\end{tabular}

\section{DISCUSSION}

There is now concensus that new phylogenies constructed using a variety of data and powerful computer software more closely reflect evolutionary history than previous phylogenies. They are proving to be useful in a variety of studies in comparative biology. The results reported here demonstrate that they can also be useful to studies of soil to plant transfer of radiouclides. The results are most rigorous at the higher taxonomic levels where statistical certainty is greatest. At lower taxonomic levels the databases are often taxonomically biased, i.e. certainly groups are well represented in the data whilst others are poorly represented. The databases have, however, already enabled the identification of certain orders of plants that have unusual radionuclide uptake characteristics. In particular, the Poales is relatively low in uptake of radionuclides when compared to the eudicots. This may not be the case for all radionuclides but the data suggest it is a widespread pattern. It is important to remember that the data analysed here is only for root uptake. Hence, if a plant species in the Poales had a high foliar interception rate for a radionuclide it might still be able to get a high radionuclide burden in total under particular circumstances. The Caryophyllales and Cucurbitales have the highest uptake of radionuclides or the oreders included in our databases. The Caryphllales includes important crop plants such as the beets, buckwheat and rhubarb together with some ecologically important fmilies such as the carnation and cacti: families. The Cucurbitales includes the gourd/melon family.

The establishment of phylogenetic effects on plant uptake has a number of consequences for discussions of soil to plant transfer of radionclides. First, the phylogenetic constraints enable general predictions of relative radionuclide concentrations in plants to be made. This is useful in circumstances where data on the uptake of a contaminated plant does not exist. At present, there is much data on transfer of radionuclides from soil to plant for important crop plants. There is much less data, however, on uptake by minor crop plants that can be important to particular human populations. Further, for many wild plant taxa there is very little data at all on which to estimate potential impacts of radionuclides on terrestrial foodchains. The establishment of some fundamental phylogenetic patterns of plant uptake of radionuclides might enable quick general predictions to be made of soil to plant transfer in those instance: in which data does not currently exist. Of course, soil availability is a key variable in determining soil to plant transfer. It might be interesting to attempt to integrate the patterns we have identified into models that cater for soil availability to assess whether or not improved predictions of soil to plant transfer can be made. 
Second, these phylogenetic patterns can be used to focus research into the mechanisms that control radionuclide uptake by plants. For example, it has been suggested that ${ }^{137} \mathrm{Cs}$ uptake into plants is primarily through Voltage Independent Cation channels in root membranes [6]. Our research has supported this suggestion by demonstrating the irrelevance of other potential transporter proteins to ${ }^{137} \mathrm{Cs}$ uptake [7]. The results reported here suggest that high ${ }^{137} \mathrm{Cs}$ uptake in the Caryophyllales might arise from VIC channel characteristics. They also sugest that the role of VIC channels in the uptake of other radionuclides may be worth investigating. The identification of mechanisms that control uptake of radionuclides might be very useful in the modification of plant uptake of radionuclides by means of conventional breeding or recombinant DNA technologies.

Third, these variations in radionuclide uptake by plants suggest that there is much biodiversity in uptake characteristics and indicate in which taxonomic groups it occurs. There is much current interest in the use of plants to extract contaminants from soils. From the data reported here a pattern is beginning to emerge indicating which plant taxa might be most useful if this decontamination strategy is to be attempted for radionuclides. Further, there is much interest in plants that do not take up radionuclides to any great extent because they might be useful for growing on contaminated soils. Again, the data reported here are beginning to suggest which groups of plants are most likely to have these characteristics.

The data reported here establish that there are some phylogenetic effects that might be important in determining soil to plant transfer of radionuclides. The variation found between taxa suggest that phylogenetic effects might be an important component of variation in soil to plant transfer of radionuclides. They also suggest a number of areas of research which might prove fruitful in understanding soil to plant transfer of radionuclides.

\section{Acknowledgements}

The authors are grateful to Dr M. Broadley (HRI, Wellesbourne, UK) for useful discussions.

\section{References.}

[1] Angiosperm Phylogeny Group. Ann Missouri. Bot. Gard., 85 (1998) 531-553.

[2] Soltis, P.S., Soltis, D.E. and Chase, M.W. Nature 402 (1999) 402-404.

[3] Doyle, J.J. Trends Plant Sci. 3 (1998) 473-478.

[4] Broadley, M.R., Willey, N.J., Wilkins, J., White P.J. Meade, A. and Baker A.J.M. New Phytol 152 (2001) 101-115

[5] Broadley M.R., Willey N.J. and Meade A. Env. Poll. 109 (1999) 341-349.

[6] White P.J. and Broadley M.R. New Phytol 147 (2000) 241-256.

[7] Broadley M.R., Bowen, H., Willey N.J. and White P.J. J. Exp. Bot. 52 (2001) 839-847 